\title{
Consistency of the decision-maker in pair-wise comparisons
}

\author{
József Temesi \\ Department of Operations Research, \\ Corvinus University Budapest, \\ Fővám tér 8, Budapest H-1093, Hungary \\ E-mail: jozsef.temesi@uni-corvinus.hu
}

\begin{abstract}
Most authors assume that the natural behaviour of the decision-maker is being inconsistent. This paper investigates the main sources of inconsistency and analyses methods for reducing or eliminating inconsistency. Decision support systems can contain interactive modules for that purpose. In a system with consistency control, there are three stages. First, consistency should be checked: a consistency measure is needed. Secondly, approval or rejection has to be decided: a threshold value of inconsistency measure is needed. Finally, if inconsistency is 'high', corrections have to be made: an inconsistency reducing method is needed. This paper reviews the difficulties in all stages. An entirely different approach is to elaborate a decision support system in order to force the decision-maker to give consistent values in each step of answering pair-wise comparison questions. An interactive questioning procedure resulting in consistent (sub) matrices has been demonstrated.
\end{abstract}

Keywords: pair-wise comparison; consistency; decision support; interactive methods.

Reference to this paper should be made as follows: Temesi, J. (2006) 'Consistency of decision-maker in pair-wise comparisons', Int. J. Management and Decision Making, Vol. 7, Nos. 2/3, pp.267-274.

Biographical notes: Dr. József Temesi received his Master's Degree in Economics from the University of Economic Sciences in Budapest, Hungary, and obtained a $\mathrm{PhD}$ in Operations Research from the Hungarian Academy of Science. Currently he is the Head of the Department of Operations Research at the Corvinus University of Budapest, where he has been a Faculty member from 1974. He was a Visiting Scholar at Northwestern University, Chicago in 1987, and spent a semester at the University of New Hampshire, as a Fulbright Professor, in 1994. Temesi served in various management positions; he was the Dean of the Faculty of Economics in the early 1990s, and from 1995 to 2004 he has been responsible for academic and scientific affairs as the Vice-Rector of the University.

\section{Introduction}

In several decision-making problems, the decision-maker (DM) estimates unknown weights of criteria or preference values of certain alternatives with respect to a given criterion using the method of pair-wise comparisons. If $w_{1}, w_{2}, \ldots, w_{n}$ are the implicit 
weights, and the DM's evaluations for every pair are $a_{i j}$ (where $a_{i j}>0$, and $a_{i j}=1 / a_{j i}$ ), then $A$ is a positive, consistent, and reciprocal matrix if $a_{i j} a_{j k}=a_{i k}$ for each $i, j, k=1, \ldots, n$, and the rank of $A$ is 1 . The usual assumption is that the DM is not consistent, and in real cases the values of $a_{i j}$ and $w_{i} / w_{j}$ differ from each other.

One of the major questions is does inconsistency really describe the 'natural behaviour' of the decision-maker? Talking about a famous example of Saaty (1980), the 'wealth of nations', Blankmeyer (1987) says: "Given an unambiguous definition of wealth and given accurate data, consistency adjustment becomes an evaluation of the respondent's expertise ...". In a case where we expect that the DM reveals his expertise through pair-wise comparisons, finding a consistent (or near-consistent) matrix could be an obvious approach.

'However, interest often centres not on objective data, but the respondent's own preferences or opinions, which may involve inconsistencies...'. Agreeing with Blankmeyer we will make that distinction, and we will allow inconsistencies in a case, where the DM is uncertain when we force him to express his preferences with the help of pair-wise comparisons. However, even if the case is that the DM is subjective, and inexperienced at expressing preferences, inconsistencies can be tried to be avoided.

After a questioning procedure it is found in both cases that the pair-wise comparison matrix is not consistent. Having an inconsistency measure, one option is to reduce inconsistency. Unfortunately, there is no unique inconsistency measure: we will list a few of them in Section 3.2. The same is true for an inconsistency reducing method. After the estimation of $w_{i j}$ (there are several methods for that problem, too), we can decide on producing a new pair-wise comparison matrix with less inconsistency. There are different concepts of doing that task, too.

\section{Sources of inconsistency}

\subsection{The decision-maker}

First we have to define the consistency of the decision-maker. The DM is absolute consistent if the rank of the pair-wise comparison matrix is 1 . In a real case, consistency means that the DM's pair-wise comparison matrix is as close to the one rank matrix as possible (near-consistency).

As outlined earlier, there is a difference between a naïve decision maker and an expert. An expert should be absolute consistent or near consistent. If it is found that the given matrix was not consistent, we have to go back to the DM. In the case of a naïve decision-maker, absolute or near-consistency is not required. It is assumed, that inconsistency comes from the DM's inability to express perfect preferences, and one has to 'intervene' only if the inconsistency is too 'high'. So inconsistency has to be measured to give a value (threshold), to accept, or to reject the given pair-wise comparison matrix.

\subsection{The method}

It is also possible that there is a built-in inconsistency in a method. If the construction of a pair-wise comparison matrix uses fixed scale to express the DM's comparative values, then inconsistency is very probable. In constructing the pair-wise comparison matrix, the most popular method is to ask the DM to compare the pairs of alternatives (criteria) in a 
scale from 1 to 9 . It is easy to see that the condition of having pairs with the property of $a_{i j} a_{j k}=a_{i k}$ for each $i, j, k=1, \ldots, n$, could not be met in quite simple cases while using that scale. Several authors mentioned this problem when they analysed the AHP method (Barzilai, 1997; Murphy, 1993; Monsuur, 1996).

The critiques of scale $(1,9)$ focus on the threshold value of the inconsistency measure used by Saaty. It has to be emphasised that the threshold problem is important, but the built-in inconsistency leads to an other, more relevant problem. If the adjustment of the pair-wise comparison matrix is to be decided, the different impacts have to be separated in order to find the element(s) to be adjusted. In a real-world decision problem our job is not to find a consistent or near consistent matrix, but to find the appropriate one. An adjustment method where a clear interpretation of inconsistency exists, and a good starting point to the DM in the revision of his preferences can be given, should be constructed.

\section{Measuring inconsistency}

\subsection{Estimation methods}

Pairwise comparison came to the forefront of the theory and applications after the pioneering work of Saaty (1980). Saaty used the eigenvector method to derive weights from a pair-wise comparison matrix:

$$
A w=\lambda w
$$

Other researchers argued that distance-minimising methods can overcome some difficulties of the AHP. Golany and Kress (1993) give a comparison of the so-called Eigenvector methods and Extremal methods. The most popular Eigenvector Method is the AHP, and a variant of this method is the Modified Eigenvector Method (Cogger and $\mathrm{Yu}, 1985)$. The most often proposed extremal methods are:

Least squares method (LSM)

$$
\min \sum_{i=1}^{n} \sum_{j=1}^{n}\left(a_{i j}-\frac{w_{i}}{w_{j}}\right)^{2}
$$

Weighted least squares method (WLSM)

$$
\min \sum_{i=1}^{n} \sum_{j=1}^{n}\left(a_{i j} w_{j}-w_{i}\right)^{2}
$$

Logarithmic least squares method (LLSM)

$$
\min \sum_{i=1}^{n} \sum_{j=1}^{n}\left(\log a_{i j}-\log w_{i}+\log w_{j}\right)^{2}
$$

Chi-square method (CSM)

$$
\min \sum_{i=1}^{n} \sum_{j=1}^{n}\left(a_{i j}-\frac{w_{i}}{w_{j}}\right)^{2}\left(\frac{w_{j}}{w_{i}}\right)
$$




$$
\sum_{i=1}^{n} w_{i}=1, \quad w_{i}>0, \quad i=1, \ldots, n
$$

There is no unified approach to compare the methods - different authors use different criteria. Barzilai (1997), for example states that LLSM is more efficient than the EM. Blankmeyer (1987) argues that WLSM is the most appropriate model. Golany and Kress (1993) introduced several criteria:

- $\quad$ minimum violations (sums up all the violations - or element preference reversals - associated with the weight vector $w$ for any given solution technique)

- total deviation (a quadratic error measure, which evaluates the sum of deviations between ratios of weights and their corresponding entry in the matrix $A$ )

- conformity (a deviation between the weight vector obtained by a particular method and an average vector across all the methods)

- robustness (the sensitivity of the solution methods to small changes in the input data)

- $\quad$ alternative solutions

- computation time

- invariance to transposition

They found that there is no dominant method (only MEV proved ineffective).

\subsection{Inconsistency measures, threshold of inconsistency}

In the case of EM methods a measure of inconsistency is needed. A positive reciprocal matrix is consistent iff $\lambda_{\max }=n$. Saaty uses the difference $\left(\lambda_{\max }-n\right)$ to create a consistency measure. His consistency ratio compares $\left(\lambda_{\max }-n\right) /(n-1)-$ the consistency index, $C I-$, with an index calculated from the same values from randomly generated reciprocal matrices, $R I$.

Saaty says that if $C I / R I>0.1$, the DM should revise his judgments on preferences. That type of threshold does not take into account the various decision situations and does not give clear interpretation of inconsistency, moreover, the $10 \%$ threshold value depends on the scale, which is being used (Monsuur, 1996).

Gass and Rapcsák (2004) use the singular value decomposition (SVD) to determine the weights They suggest the Frobenius norm of the difference between the original pair-wise comparison matrix and one formed by the SVD determined weights as an inconsistency measure. The SVD approach and EM give the same result in the case of positive and consistent matrices, the differences are in the inconsistent cases. As the authors argue that the SVD approach has much to offer as it stems directly from rank one considerations, and the associated Frobenius norm appears to be an appropriate metric that may be adaptable as an inconsistency measure.

\subsection{How to improve consistency}

Most of the authors agree that improving consistency should start with the element where $\left|a_{i j}-w_{i} / w_{j}\right|$ is maximal. However, the measure of consistency index based on the largest 
eigenvalue does not give any hint to say which matrix element contributed to the increase of inconsistency. Koczkodaj (1993) developed a new consistency measure to locate the inconsistency. His measure is based on the matrix elements, and therefore it can be applied to find a plausible starting point for the improving procedure.

As Koczkodaj says, his "consistency measure is always associated with a certain element and it is not an enigmatic global matrix characteristic like an eigenvalue". However, some researchers are seeking global ways to increase consistency of a given matrix. Xu and Wei (1999) developed an algorithm where a new, more consistent matrix can be generated with better maximum eigenvalue and with the same priority vector.

As it is seen, one of the crucial issues in consistency improvement is whether we make a correction in one matrix element only, or are we trying to adjust all (or most of) the elements. The philosophy of the latter approach is that the DM's inconsistency caused a kind of perturbation compared to the 'proper' matrix, and with the adjustment method it is being built up in a balanced way, while increasing consistency. The main drawback of this procedure is - based on real-world applications - that the new pair-wise comparison matrix is sometimes very artificial in the eyes of the DMs, and they have serious difficulties to compare it to their original pair-wise evaluations. Modifying one matrix element in each step proved much more DM-friendly.

Such argumentation leads to the other crucial question: do we need the personal participation of the DM in the improvement procedure? There are cases, of course, when it is impossible to turn to the DM again. In textbook examples the authors would like to avoid repetition of the questioning procedure, stating that the DM is not available for a repeated procedure, or another questioning (mainly in the case of larger matrices) would lead to a confused situation. However, successful real applications mostly require the presence of the decision maker(s).

In our approach, the decision problem and the decision maker together create the decision environment. Interactive and not interactive cases should be separated, and in the interactive decision support system a learning module is also needed -the opportunity to the decision-maker to learn what is consistency and how to improve consistency should be given.

\section{Questioning procedures}

In a real-world decision problem, the focus is not on the approximation method, but on the questioning procedure. When having an inexperienced decision-maker, he should first be taught the method of pair-wise comparisons (because it is easy to understand and is intuitively appealing), and in a second step he has to be shown the nature of weights derived from a pair-wise comparison matrix. Solving his problem could be done after this 'tutorial' only.

\subsection{Systems with consistency control}

Step 1: Checking consistency. The prerequisite of the execution of this step is to have a consistency measure. As we outlined earlier, there are several approaches to construct a consistency measure (consistency index). In case of absolute consistency there is no need to modify the pair-wise comparisons, the procedure is over, weights are obtained. If inconsistency occurs, we have to go to 
Step 2: Accepting the level of inconsistency. The prerequisite of the execution of this step is to have a threshold value. As outlined earlier, a natural threshold value does not exist. In case of having a better inconsistency value than the threshold, the procedure is over, we accept the given comparison matrix and the weights obtained from that matrix. If inconsistency is higher than the threshold value, we have to go to Step 3.

If the consistency measure has the property that lesser values mean less consistency, then even if we do not have a threshold value, we can decide on terminating the procedure (time-limit, high cost could effect that decision, or the DM itself can stop the iteration).

Step 3: Modifying the pair-wise comparison matrix. The prerequisite of the execution of this step is to have an improvement method. After executing the method we go back to Step 1.

\subsubsection{Maximum deviation}

We can offer the DM to make correction in his judgment related to the maximum absolute deviation between $a_{i j}$ and $w_{i} / w_{j}$. Most of the existing software packages apply that criterion.

\subsubsection{Using basic reciprocal matrices}

A basic reciprocal matrix has three comparisons and it can be reduced to a vector of three coordinates $[a, b, c]$ (Koczkodaj, 1993). The matrix is consistent if $b=a \times c$. With the help of the vectors, $[b / a, b, c],[a, a \times c, c]$ and $[a, b, b / a]$, an inconsistency measure can be defined as the relative distance to the nearest consistent basic reciprocal matrix, represented by one of these three vectors for a given metric. This definition can be extended to matrices of higher orders: for a given matrix element it can be defined as maximum of the consistency measure of all possible triads, which include this element.

The author suggests that only a fixed number of changes may be allowed to avoid 'a race for total consistency'.

\subsubsection{Random questioning}

The full matrix with different (randomly selected) sequences of pair-wise comparisons can be obtained. For a naïve decision-maker, who is uncertain in the ratios (or verbal statements), the different sequences will result in different matrices. There could be various approaches to find a matrix representing the 'true values' of the DM's preferences. This particular method could be a part of the learning process: the DM can learn more about the problem itself (alternatives or criteria).

It is possible to aggregate his opinions - the problem of any averaging procedure could be that the elements are not discussed with the decision maker. In a modified version of the aggregation-based procedures the DM has the possibility to evaluate the matrix and to modify certain elements. 


\subsubsection{Using intervals}

In a (random) questioning procedure, after a fixed number of comparisons, intervals for the further evaluations can be given, using the $a_{i j} a_{j k}=a_{i k}$ equalities for each $i, j, k=1, \ldots, n$. The decision-maker can choose any value in the interval, if he would like to be in harmony with prior comparisons.

\subsection{Decision-aiding}

Having an inconsistent pair-wise comparison matrix, we can go back to the decision-maker to make the choices more rational. That simple question and answer procedure can also be applied as a learning tool: the DM can learn the meaning of consistency, based on his own problem.

In a small example the following pair-wise comparison matrix was given by the DM:

$$
\left[\begin{array}{ccc}
1 & 2 & 7 \\
1 / 2 & 1 & 9 \\
1 / 7 & 1 / 9 & 1
\end{array}\right]
$$

$Q_{1}$ : If $a_{23}$ is reduced to 3.5 , your answers are consistent. Do you accept it? If not, what would be your estimation for $a_{23}$ ?

$A_{1}$ : $\quad$ I do not want to reduce $a_{23}$.

Value of $\mathbf{a}_{23}=\mathbf{9}$.

$Q_{12}$ : In case of consistency $a_{12}=7 / 9$. What is your value for that comparison?

$A_{12}$ : The value of $a_{12}$ should be 1 .

Value of $\mathbf{a}_{12}=\mathbf{1}$ and we calculate $a_{13}=a_{12} \times a_{23}, \mathbf{a}_{13}=\mathbf{9}$.

The new matrix is:

$$
\left[\begin{array}{ccc}
1 & 1 & 9 \\
1 & 1 & 9 \\
1 / 9 & 1 / 9 & 1
\end{array}\right]
$$

Let suppose, that the answer for $Q_{l}$ is different:

$A_{2}$ : $\quad$ I would give the value $a_{23}=6$.

Value of $\mathbf{a}_{23}=\mathbf{6}$.

$Q_{22}$ : $\quad$ In case of consistency $\mathrm{a}_{12}=7 / 6$. What is your comparative value?

$A_{22}$ : My comparison is 1.5 .

Value of $\mathbf{a}_{\mathbf{1 2}}=\mathbf{3} / \mathbf{2}$ and we calculate $a_{13}=a_{12} \times a_{23}, \mathbf{a}_{13}=\mathbf{9}$.

The new matrix is:

$$
\left[\begin{array}{ccc}
1 & 3 / 2 & 9 \\
2 / 3 & 1 & 6 \\
1 / 9 & 1 / 6 & 1
\end{array}\right]
$$




\section{Conclusion}

In a real-world decision problem, the DM is interested in learning his preferences and revealing them with a satisfying result. The pair-wise comparison matrix is a very convenient and reliable tool for that purpose. However, most of the existing methods focus on the estimation problem of a given pair-wise comparison matrix, and do not allow an interaction with the DM. Decision support systems have to incorporate options for the DM to evaluate the consistency of the comparisons, and to change one or more elements in the matrix. The difficulty of building a DSS is that there is no unique measure of inconsistency, and it is hard to find the element from where the modification can start. The recommendation in this study is to offer more than one ways for solving the inconsistency problem, and the DM can choose based on the nature of the particular decision problem. In case of achieving absolute consistency (that could occur in specific decision problems, where the expertise of the DM has been proved) the DSS will focus on a well-structured questioning procedure.

\section{References}

Barzilai, J. (1997) 'Deriving weights from pairwise comparison matrices', Journal of Operational Research Society, Vol. 48, pp.1226-1232.

Blankmeyer, E. (1987) 'Approaches to consistency adjustment', Journal of Optimization Theory and Applications, Vol. 54, No. 3., pp.479-488.

Cogger, K.O. and Yu, P.L. (1985) 'Eigenweight vectors and least distance approximation for revealed preference in pairwise weight ratios', Journal of Optimization Theory and Applications, Vol. 46, pp.483-491.

Gass, S.I. and Rapcsák, T. (2004) 'Singular value decomposition in AHP', European Journal of Operational Research, Vol. 154, pp.573-584.

Golany, B. and Kress, M. (1993) 'A multicriteria evaluation of methods for obtaining weights from ratio-scale matrices', European Journal of Operational Research, Vol. 69, pp.210-220.

Koczkodaj, W.W. (1993) 'A new definition of consistency of pairwise comparisons', Mathl. Comput. Modelling, Vol. 18, No. 7., pp.79-84.

Monsuur, H. (1996) 'An intrinsic consistency threshold for reciprocal matrices', European Journal of Operational Research, Vol. 96, pp.387-391.

Murphy, C.K. (1993) 'Limits on the analytic hierarchy process from its inconsistency index', European Journal of Operational Research, Vol. 65, pp.138-139.

Saaty, T.L. (1980) The Analytic Hierarchy Process, McGraw Hill, New York.

Saaty, T.L. (1990) 'Eigenvector and logarithmic least squares', European Journal of Operational Research, Vol. 48, pp.156-160.

$\mathrm{Xu}, \mathrm{Z}$. and Wei, C. (1999) 'A consistency improving method in the analytic hierarchy process', European Journal of Operational Research, Vol. 116, pp.443-449. 